\title{
The relevance of health literacy to mHealth
}

\author{
Gary L. Kreps \\ Ph.D., University Distinguished Professor and Director of the Center for Health and Risk \\ Communication, George Mason University, 4400 University Drive, MS 3D6, Fairfax, VA 22030, USA \\ Tels: 703-993-1094, 202-594-2794; E-mail: gkreps@gmu.edu
}

\begin{abstract}
This paper examines the importance of health literacy to the design and use of mobile digital health information technology (mHealth) applications. Over the past two decades mHealth has evolved to become a major health communication channel for delivering health care, promoting health, and tracking health behaviors. Yet, there are serious communication challenges that must be addressed concerning the best way to design and utilize mHealth application to achieve key health promotion goals, including assuring the appropriateness and effectiveness of mHealth messaging for audiences with different communication competencies, styles, and health literacy levels, to ensure that mHealth applications are truly effective tools for health promotion.

Health literacy is one of the major communication issues relevant to the effective use of mHealth. To be effective, mhealth applications need to match the messages conveyed via these mobile media to the specific health communication needs, orientations, and competencies of intended audience members. Unfortunately, current evidence suggests that many mHealth applications are difficult for audiences to utilize because they provide health information that is not easy for many consumers to understand and apply.

Health literacy refers to the ability of participants within the health care system to accurately interpret and utilize relevant health information and resources to achieve their health goals. Evidence suggests that many consumers possess limited levels of health literacy to adequately understand health information, especially when they are feeling ill, since health literacy is both a trait (limited education, language facility, etc.), and a state condition (based on how their current physical and mental states influence their abilities to communicate effectively). Therefore, it is incumbent upon mHealth developers to design and utilize message systems. Strategies for designing and implementing mHealth applications to meet the health literacy levels of different audiences are described in this article.
\end{abstract}

Keywords: mHealth, health literacy, health communication, applications, health promotion

\section{Introduction}

The use of mHealth (mobile digital information technologies designed to deliver care, track health behaviors, and promote health) has evolved over the past two decades to become a major channel for health promotion $[17,50]$. This increasingly popular use of mHealth channels for communication has mirrored the phenomenal international growth in public adoption of mobile phone technologies, especially the use of smart phones that enable Internet access [50]. Implementation of mHealth applications has shown tremendous promise as an important evolving communication medium for supplementing and extending the use of more traditional channels for health communication, such as in-person clinical interpersonal interactions with health care providers (and other sources of health information), use of print health education materials (such as books, articles, pamphlets, and posters), public presentations (such as school and conference lectures), and access to entertainment media programs via radio, television, and film [29].

A number of different emerging and interrelated mobile communication systems have been adopted and adapted for delivering mHealth, including the increasing use of: 
- audio and video enabled telemedicine applications, such as closed circuit telephone and/or televised conferencing systems, with both data collection tools (often collecting images, or measuring body temperature, heart rate, and brain activity for diagnostic and monitoring purposes) and delivery tools (that can remotely control surgical equipment, and provide treatment instructions) can be set up in different sites to provide mediated (virtual) care to patients who may not have easy access to health care delivery systems [52];

- telephonic and internet-based message systems that can provide individuals with health information via SMS text messages, phone calls, or e-mail via mobile phones [15];

- websites and web-based portals (intranets) that are available to health care consumers via portable lap-top computers, tablets, and smart phones;

- wearable (sometimes implantable) devices such as fitness tracking wristbands and other digital monitoring devices [3];

- specialized application software programs (apps) that are typically downloaded on to smart phones, tablets, or laptop computers that provide health information and support, and are often linked to Internet delivered resources and services [10].

The added value provided by mhealth applications for health promotion is the ability to use these media to provide key audiences (health care consumers and providers) with relevant health information and support that they need exactly at the points in time when they need it and right where they need it to enhance timely and on-the-spot informed health decision making [29]. As a result, there is growing enthusiasm for using mHealth applications within health care and health promotion communities with a range of different audiences, both domestically and internationally [10,50].

Yet, there are serious communication challenges that must be addressed concerning the best uses of mHealth for health promotion, including assuring the appropriateness and effectiveness of mHealth messaging for audiences with different communication competencies, styles, and health literacy levels, to ensure that mHealth applications are truly effective tools for health promotion $[7,19]$. It is not merely the use of mobile communication channels that can make these applications effective, it is also the ability to match the messages conveyed via these mobile media to the specific health communication needs, orientations, and competencies of intended audience members [49]. For example, practitioners of mHealth must strategically develop and refine health messaging to match the health literacy levels of key audiences $[25,35]$.

This paper examines the tremendous potential of mHealth applications for providing relevant health information to diverse populations of health care providers and consumers and for enhancing health decision making. The article also illustrates how mHealth applications can be designed to deliver appropriate and effective messages that meet the unique communication competencies, styles, and health literacy levels of different key audiences to become effective health communication channels.

\section{The promise of mHealth}

There have been a number of unique and important contributions made to the delivery of care and the promotion of health with the use of mHealth applications as supplements to traditional channels of health communication [49]. For example, mHealth applications have been shown to increase the timeliness of health care delivery and the ability to provide relevant health information to key audiences wherever they may be via their mobile phones that they carry with them [26]. Health care providers like mobile health applications and have been early adopters and users of mhealth systems to gather health information and 
to communicate with their colleagues and clients [37]. mHealth systems have been successfully used to address a wide variety of serious health conditions, including heart health promotion [12], infectious disease control [21], respiratory health [46], maternal and child health [6,48].

There has also been a great deal of evidence that mHealth has been used effectively to promote consumers' adoption of healthy behaviors and reduction of unhealthy behaviors [11], including the following behavioral health issues: consumer adherence with treatment recommendations and prescribed medications [14,18], reducing risky sex behaviors and adopting safer sex strategies [23,41], increasing physical activity and exercise [9,30], maintaining healthy diets [22,47], tobacco control $[1,53]$ drug and alcohol abuse reduction [20,39,44], and promoting screening behaviors for early detection of serious health problems $[13,18,33]$. Furthermore, evidence suggests that mHealth applications have the potential to improve health care delivery performance and retention of community health workers [24].

Rapid and widespread adoption of mobile communication technologies, such as the smart phones, tablets, and wearable devices have been shown to provide health communicators with powerful new channels for disseminating health information [10,52]. Mobile communication tools have become increasingly well-liked, adopted, and frequently used channels for communication by diverse audiences, including by members of at-risk populations who often suffer from health inequities and poor access to health information [16]. mHealth application provide great opportunities to reach key audiences with health information right at the point when they are making important health decisions, providing these audiences with relevant health information exactly when they need it and where they need it [26]. Advances in mHealth technologies are making these communication tools increasingly easy to use, enable them to disseminate a variety of different message formats (text, oral, graphic, video, etc), and enable interactivity (feedback between health communicators) [10]. mHealth technologies can be integrated with other digital health tools, such as exercise trackers, telehealth monitors, and electronic health records, to provide users with rich sources of health information. Tailored information systems and the use of artificial intelligence can be used with mHealth tools to provide relevant, interactive, adaptive, and sensitive health messages to users [31].

\section{Challenges to using mHealth technologies effectively}

There are serious communication challenges that must be addressed before mHealth technologies can reach their potential as effective tools for health promotion. These challenges include designing appropriate and effective mHealth messaging for use with audiences who possess different communication competencies, styles and health literacy levels $[29,34,36]$. While sophisticated and often elegant new technological media receive the bulk of attention in mHealth, the effects of these media on health promotion are highly dependent on the quality of messages that are exchanged via these advanced media.

Health literacy refers to the ability of participants within the health care system to accurately interpret and utilize relevant health information and resources to achieve their health goals [5]. Evidence suggests that many consumers possess limited levels of health literacy to adequately understand health information, especially when they are feeling ill, since health literacy is both a trait (limited education, language facility, etc.), and a state condition (based on how their current physical and mental states influence their abilities to communicate effectively) [5]. Therefore, it is incumbent upon mHealth developers to design and utilize message systems that will be easy for consumers with health literacy challenges to understand [40].

Due to the limited channel capacity on many mHealth technologies, such as the limited number of characters that can be used for SMS texting or tweeting, care must be taken to strategically design 
health messages that will convey full, accurate, timely health information, as well as resonate with and make sense to diverse audiences [31]. In many cases this will mean targeting, or even tailoring, health messages for specific users so the messages include appropriate language, provide meaningful examples, and suggest health options that the users can implement.

Whenever possible, it is preferable to build in feedback loops into mHealth systems, so users can pose questions and receive clarifications [31]. If possible, mHealth systems should be designed to incorporate visual, animated, and narrative messages to enhance understanding, especially for users with low health literacy and numeracy skills [31]. Narrative descriptions of health topics that imbed health information within rich, dramatic, and engaging stories has been found to be a particularly robust communication strategy for communicating health information to users with low levels of health literacy.

In addition, Weinstein et al., 2014 [52], describe three major bureaucratic challenges that challenge the widespread adoption and effective use of telemedicine and mHealth applications:

- The need for clear and standardized reimbursement strategies and business models to make it less challenging to fund the development, implementation, and maintenance of mHealth interventions;

- The need to establish interstate medical licensure for implementing domestic mHealth applications that cross state lines, as well as international licensing for applications that cross national boundaries;

- The need for hospital credentialing to implement mHealth applications as standardized tools within modern health care delivery systems.

\section{Evaluating mHealth applications}

Message design processes for mHealth technologies should begin with careful audience analysis of potential system users, identifying key communication factors, including language use, health literacy levels, communication channel utilization patterns, familiarity with mHealth technologies, interest levels in different health topics, relevant health beliefs, and attitudes [19,42]. The Centers for Disease Control and Prevention (CDC) recently introduced an excellent tool for evaluating the health literacy levels of health promotion materials, the CDC's Clear Communication Index, that can be easily applied to the evaluation of mHealth messaging [2]. Similarly, Stoyanov and colleagues [51] recently introduced and tested the Mobile App Rating Scale (MARS), demonstrating that is a simple, objective, and reliable measuring instrument for designing effective mHealth applications.

With key background information from evaluation research mHealth developers can guide the design of appropriate, understandable, relevant, and compelling health messages to deliver via mHealth devices [4]. Message testing with targeted audiences can also provide relevant information about how well potential mHealth users can understand and accept health messages [36]. Additionally, usability testing of mHealth technologies and information programs can provide relevant information for refining mHealth systems [38].

In addition, it is important to assess the economic viability of mHealth applications to ensure that these health promotion tools are cost-effective for different users and in different settings $[8,45]$. Cost and benefit analyses can provide information needed to demonstrate the value of mHealth applications, support decision making about adoption and institutionalization of these applications, and also provide direction for developing business models for supporting the introduction and continued use of these new health promotion systems $[28,45]$. Bergmo provides cogent advice about conducting relevant economic 
analyses of ehealth systems that can be effectively applied to assessing the costs and benefits of mHealth applications [8].

\section{Conclusion}

Mounting evidence clearly illustrates that mHealth applications have tremendous potential for supplementing traditional channels of health communication as an important evolving communication medium for promoting health $[16,17,50,52,54]$. The ability of mHealth systems to provide individuals with the relevant health information they need when they need it and where they need it has great potential for enhancing health decision making. Yet, there are serious communication challenges that must be addressed, including the appropriateness and effectiveness of mHealth messaging for audiences with different communication competencies, styles and health literacy levels, before mHealth can become an effective tool for health promotion [29]. This article provides recommendations for the development and use of mHealth technologies to meet the unique health communication needs of diverse users for enhancing health decision making and improving health outcomes.

A critical factor in the whether mhealth applications will reach their potential to make significant contributions to the promotion of public health depends on the ability of these systems to communicate effectively with different audiences, who have different health information needs and unique communication orientations and capacities [27,31]. There are a broad range of different potential users for mhealth systems who will want these systems to communicate effectively with them, providing them with the specific information they need and using language and examples that they can relate to [3,27]. For example, research shows that mHealth systems have been designed for older and younger health care consumers, users with different education levels, people with different sexual orientations, people with drug and alcohol addictions, people from different minority and ethnic groups, people from different countries, including those who speak different languages, all of whom are likely to have different communication preferences and skills $[14,20-23,30,44,48]$. It will be important for mHealth system designers to conduct audience analysis research to evaluate the literacy levels of different mHealth users to determine the best ways to communicate effectively with these groups [2,3].

Adapting to the health literacy levels of different mHealth users is one of the most critical communication factors that will influence the effectiveness of mHealth in effectively reaching and influencing system users $[2,5,34,36]$. Adapting to differing levels of health literacy means that the messaging used in mHealth systems will need to be designed specifically for different groups of users, assuring the utilization of appropriate language, examples, and visuals that are meaningful for these groups of users. The most current mHealth systems are now being designed to interactively assess the communication competencies, needs, expectations, and literacy levels of different system users. These modern mHealth systems are programmed with smart, adaptive, interactive learning systems that utilize artificial intelligence to learn about and adjust messages to individual users $[32,43]$. As these smart mHealth systems interact with users they learn through analysis of user comments and responses to system messages how to best adapt future system messages for these users, enabling the use of relationally sensitive and appropriate communication that can enhance communicative understanding, relevance, and immediacy for users of mHealth systems [32]. 


\section{References}

[1] L.C. Abroms, M. Ahuja, Y. Kodl, L. Thaweethai, J. Sims, J.P. Winickoff and R.A. Windsor, Text2Quit: Results from a pilot test of a personalized, interactive mobile health smoking cessation program, Journal of Health Communication 17(suppl 1) (2012), 44-53. doi:10.1080/10810730.2011.649159.

[2] J.M. Alpert, L. Desens, A.H. Krist, R.A. Aycock and G.L. Kreps, Measuring health literacy levels of a patient portal using the CDC's Clear Communication Index. Health promotion practice, 2016. doi:10.1177/1524839916643703.

[3] J.M. Alpert, A.H. Krist, B.A. Aycock and G.L. Kreps, Designing user-centric patient portals: Clinician and patients' uses and gratifications. Telemedicine and e-Health, 2016 ahead of print. doi:10.1089/tmj.2016.0096.

[4] J.M. Alpert, A.H. Krist, R.A. Aycock and G.L. Kreps, Applying multiple methods to comprehensively evaluate a patient portal's effectiveness to convey information to patients, Journal of Medical Internet Research 18(5) (2016), e112. doi:10. 2196/jmir.5451.

[5] J. Amann, S. Rubinelli and G.L. Kreps, Revisiting the concept of health literacy. The patient as information seeker and provider, European Health Psychologist 17(6) (2015), 286-290. doi:10.3390/ijerph6020492.

[6] M. Amoakoh-Coleman, A.B. Borgstein, S.F. Sondaal, D.E. Grobbee, A.S. Miltenburg, M. Verwijs, E.K. Ansah, J.L. Browne and K. Klipstein-Grobusch, Effectiveness of mHealth interventions targeting health care workers to improve pregnancy outcomes in low-and middle-income countries: A systematic review, Journal of Medical Internet Research 18(8), (2016). doi:10.2196/jmir.5533.

[7] T.D. Aungst, K.A. Clauson, S. Misra, T.L. Lewis and I. Husain, How to identify, assess and utilise mobile medical applications in clinical practice, International Journal of Clinical Practice 68(2) (2014), 155-162. doi:10.1111/ijcp.12375.

[8] T.S. Bergmo, How to measure costs and benefits of eHealth interventions: An overview of methods and frameworks, Journal of Medical Internet Research, 2015. doi:10.2196/jmir.4521.

[9] K.C. Blackman, J. Zoellner, L.M. Berrey, R. Alexander, J. Fanning, J.L. Hill and P.A. Estabrooks, Assessing the internal and external validity of mobile health physical activity promotion interventions: A systematic literature review using the RE-AIM framework, Journal of Medical Internet Research 15(10) (2013), e224. doi:10.2196/jmir.2745.

[10] E. Boudreaux, M. Waring, R. Hayes, R. Sadasivam, S. Mullen and S. Pagoto, Evaluating and selecting mobile health apps: Strategies for healthcare providers and healthcare organizations, Translational Behavioral Medicine 4(4) (2014), 363-371. doi:10.1007/s13142-014-0293-9.

[11] D.B. Buller and A.H.L. Floyd, Internet-based interventions for health behavior change, in: EHealth Applications: Promising Strategies for Behavior Change, S. Noar and N.W. Harrington, eds, Routledge, New York, 2012, pp. 59-78.

[12] L.E. Burke, J. Ma, K.M. Azar, G.G. Bennett, E.D. Peterson, Y. Zheng, W. Riley, J. Stephens, S.H. Shah, B. Suffoletto and T.N. Turan, Current science on consumer use of mobile health for cardiovascular disease prevention a scientific statement from the American Heart Association, Circulation 132(12) (2015), 1157-1213. doi:10.1161/CIR.0000000000000232.

[13] C. Catalani, W. Philbrick, H. Fraser, P. Mechael and D.M. Israelski, mHealth for HIV treatment \& prevention: A systematic review of the literature, Open AIDS J 7 (2013), 17-41. doi:10.1371/journal.pmed.1001363.

[14] B.H. Chi and J.S. Stringer, Effects of a mobile short message service on antiretroviral treatment adherence in Kenya (WelTel Kenya1): A randomized trial, Lancet 376(9755) (2010), 1807-1808. doi:10.1016/S0140-6736(10)62046-6.

[15] C. Déglise, L.S. Suggs and P. Odermatt, SMS for disease control in developing countries: A systematic review of mobile health applications, Journal of Telemedicine and Telecare 18(5) (2012), 273-281. doi:10.1258/jtt.2012.110810.

[16] M. Fiordelli, N. Diviani and P.J. Schulz, Mapping mHealth research: A decade of evolution, Journal of Medical Internet Research 15(5) (2013), e95. doi:10.2196/jmir.2430.

[17] S. Fox and D.M. Duggan, Mobile health 2012, Pew Internet Project. November 8, 2012.

[18] C. Free, G. Phillips, L. Galli, L. Watson, L. Felix, P. Edwards, V. Patel and A. Haines, The effectiveness of mobile-health technology-based health behaviour change or disease management interventions for health care consumers: A systematic review, PLoS Med 10(1) (2013), e1001362. doi:10.1371/journal.pmed.1001362.

[19] Q.H. Grundy, Z. Wang and L.A. Bero, Challenges in assessing mobile health app quality: A systematic review of prevalent and innovative methods, American Journal of Preventive Medicine, 2016. doi:10.1016/j.amepre.2016.07.009.

[20] D.H. Gustafson, F.M. McTavish, M.Y. Chih, A.K. Atwood, R.A. Johnson, M.G. Boyle, M.S. Levy, H. Driscoll, S.M. Chisholm, L. Dillenburg and A. Isham, A smartphone application to support recovery from alcoholism: A randomized clinical trial, JAMA Psychiatry 71 (2014), 566-572. doi:10.1001/jamapsychiatry.2013.4642.

[21] Y.P. Ha, M.A. Tesfalul, R. Littman-Quinn, C. Antwi, R.S. Green, T.O. Mapila, S.L. Bellamy, R.T. Ncube, K. Mugisha, A.R. Ho-Foster and A.A. Luberti, Evaluation of a mobile mealth approach to tuberculosis contact tracing in Botswana, Journal of Health Communication 26 (2016), 1-7. doi:10.1080/10810730.2016.1222035.

[22] L. Hebden, A. Cook, H.P. Ploeg, L. King, A. Bauman and M. Allman-Farinelli, A mobile health intervention for weight management among young adults: A pilot randomised controlled trial, Journal of Human Nutrition and Dietetics 27(4) (2014), 322-332. doi:10.1111/jhn.12155. 
[23] I.W. Holloway, E. Rice, J. Gibbs, H. Winetrobe, S. Dunlap and H. Rhoades, Acceptability of smartphone applicationbased HIV prevention among young men who have sex with men, AIDS and Behavior 18(2) (2014), 285-296. doi:10. 1007/s10461-013-0671-1.

[24] K. Källander, J.K. Tibenderana, O.J. Akpogheneta et al., Mobile Health (mHealth) approaches and lessons for increased performance and retention of community health workers in low- and middle-income countries: A review, Journal of Medical Internet Research 15(1) (2013), e17. doi:10.2196/jmir.2130.

[25] H. Kim and B. Xie, Health literacy and Internet- and mobile app-based health services: A systematic review of the literature, Proceedings of the Association for Information Science and Technology 52(1), 1-4. doi:10.1002/pra2.2015. 145052010075.

[26] P. Klasnja and W. Pratt, Healthcare in the pocket: Mapping the space of mobile-phone health interventions, Journal of Biomedical Informatics 45(1) (2012), 184-198. doi:10.1016/j.jbi.2011.08.017.

[27] G.L. Kreps, One size does not fit all: Adapting communication to the needs and literacy levels of individuals, Annals of Family Medicine (2006), http://www.annfammed.org/cgi/eletters/4/3/205. doi:10.1370/afm.534.

[28] G.L. Kreps, Evaluating health communication programs to enhance health care and health promotion, Journal of Health Communication 19(2) (2014), 1449-1459. doi:10.1080/10810730.2014.954080.

[29] G.L. Kreps, Communication technology and health: The advent of ehealth applications, in: Communication and Technology, L. Cantoni and J.A. Danowski, eds, De Gruyter Mouton Publications, Berlin, Germany, 2015, pp. 483-493.

[30] G.L. Kreps, S. Joy, M. Cary, H. Wolf, M. Villagran, X. Cai and X. Zhao, Evaluating the use of mobile health information technology to promote physical activity and weight management for addressing the obesity epidemic, Washington, DC, 2010, Presented to the MHealth Summit.

[31] G.L. Kreps and L. Neuhauser, Artificial intelligence and immediacy: Designing health communication to personally engage consumers and providers, Patient Education and Counseling 92(2) (2013), 205-210. doi:10.1016/j.pec.2013.04. 014 .

[32] G.L. Kreps and L. Neuhauser, Artificial intelligence and immediacy: Designing health communication to personally engage consumers and providers, Patient Education and Counseling 92 (2013), 205-210. doi:10.1016/j.pec.2013.04.014.

[33] H.Y. Lee, J.S. Koopmeiners, T.G. Rhee, V.H. Raveis and J.S. Ahluwalia, Mobile phone text messaging intervention for cervical cancer screening, Obstetrical \& Gynecological Survey 70(1) (2015), 26-27. doi:10.1097/OGX.0000000000000142.

[34] H. Levy and A. Janke, Health literacy and access to care, Journal of Health Communication 21(suppl 1) (2016), 43-50. doi:10.1080/10810730.2015.1131776.

[35] H. Levy, A.T. Janke and K.M. Langa, Health literacy and the digital divide among older americans, Journal of General Internal Medicine 30(3) (2015), 284-289. doi:10.1007/s11606-014-3069-5.

[36] H. Levy, A.T. Janke and K.M. Langa, Health literacy and the digital divide among older americans, Journal of General Internal Medicine 30(3) (2015), 284-289. doi:10.1007/s11606-014-3069-5.

[37] J. Li, A. Talaei-Khoei, H. Seale, P. Ray and C.R. MacIntyre, Health care provider adoption of eHealth: Systematic literature review, Interactive Journal of Medical Research 2(1) (2013), e7. doi:10.2196/ijmr.2468.

[38] X. Ma, B. Yan, G. Chen, C. Zhang, K. Huang, J. Drury and L. Wang, Design and implementation of a toolkit for usability testing of mobile apps, Mobile Networks and Applications 18(1) (2013), 81-97. doi:10.1007/s11036-012-0421-z.

[39] M.L. Mares, D.H. Gustafson, J.E. Glass, A. Quanbeck, H. McDowell, F. McTavish, A.K. Atwood, L.A. Marsch, C. Thomas, D. Shah and R. Brown, Implementing an mHealth system for substance use disorders in primary care: A mixed methods study of clinicians' initial expectations and first year experiences, BMC Medical Informatics and Decision Making 16(1) (2016), 126. doi:10.1186/s12911-016-0365-5.

[40] P. McNiel and E.C. McArthur, Evaluating health mobile apps: Information literacy in undergraduate and graduate nursing courses, Journal of Nursing Education 55(8) (2016), 480. doi:10.3928/01484834-20160715-12.

[41] L.C. Miller, P.R. Appleby, J.L. Christensen, C. Godoy, M. Si, C. Corsbie-Massay, S.J. Read, S. Marsella, A.N. Anderson and J. Klatt, Virtual interactive interventions for reducing risky sex: Adaptations, integrations, and innovations, in: EHealth Applications: Promising Strategies for Behavior Change, S. Noar and N.W. Harrington, eds, Routledge, New York, 2012, pp. 79-95.

[42] L. Neuhauser and G.L. Kreps, Integrating design science theory and methods to improve the development and evaluation of health communication programs, Journal of Health Communication 19(12) (2014), 1460-1471.

[43] L. Neuhauser, G.L. Kreps, K. Morrison, M. Athanasoulis, N. Kirienko and D. Van Brunt, Using design science and artificial intelligence to improve health communication: ChronologyMD case example, Patient Education and Counseling 2013(92) (2013), 211-217. doi:10.1016/j.pec.2013.04.006.54.

[44] M. Schulte, D. Liang, F. Wu, Y.C. Lan, W. Tsay, J. Du, M. Zhao, X. Li and Y.I. Hser, A smartphone application supporting recovery from heroin addiction: Perspectives of patients and providers in China, Taiwan, and the USA, Journal of Neuroimmune Pharmacology 4 (2016), 1-2. doi:10.1007/s11481-016-9653-1.

[45] J. Schweitzer and C. Synowiec, The economics of ehealth and mhealth, Journal of Health Communication 17S (2012), 73-81. doi:10.1080/10810730.2011.649158. 
[46] L. Seoane, J.P. Bakker, S. Bertisch, C. Pham, N. McNaughton, J. Park, K. Severensin, L.A. Bazzano and K.J. Pak, Effect of mobile health technology on positive airway pressure adherence in patients with sleep apnea, InB63. My Way: Osa Outpatient Models Of Care 2016, May (pp. A4185-A4185). American Thoracic Society. doi:10.1164/ajrccm-conference. 2016.193.1_MeetingAbstracts.A4185.

[47] B.M. Silva, J.J. Rodrigues, I. de la Torre Díez, M. López-Coronado and K. Saleem, Mobile-health a review of current state in 2015, Journal of Biomedical Informatics 31(56) (2015), 265-272. doi:10.1016/j.jbi.2015.06.003.

[48] S.F.V. Sondaal, J.L. Browne, M. Amoakoh-Coleman et al., Assessing the effect of mHealth interventions in improving maternal and neonatal care in low- and middle-income countries: A systematic review, PLoS ONE 11(5) (2016), e0154664. doi:10.1371/journal.pone.0154664.

[49] S.R. Steinhubl, E.D. Muse and E.J. Topol, Can mobile health technologies transform health care?, JAMA 310(22) (2013), 2395-2396. doi:10.1001/jama.2013.281078.

[50] S.R. Steinhubl, E.D. Muse and E.J. Topol, The emerging field of mobile health, Science Translational Medicine 7(283) (2015), 283rv3. doi:10.1126/scitranslmed.aaa3487.

[51] S.R. Stoyanov, L. Hides, D.J. Kavanagh, O. Zelenko, D. Tjondronegoro and M. Mani, Mobile app rating scale: A new tool for assessing the quality of health mobile apps, JMIR mHealth and uHealth 3(1) (2015), e27. doi:10.2196/mhealth. 3422 .

[52] R.S. Weinstein, A.M. Lopez, B.A. Joseph, K.A. Erps, M. Holcomb, G.P. Barker and E.A. Krupinski, Telemedicine telehealth and mobile health applications that work: Opportunities and barriers, The American Journal of Medicine 127(3) (2014), 183-187. doi:10.1016/j.amjmed.2013.09.032.

[53] R. Whittaker, H. McRobbie, C. Bullen, R. Borland, A. Rodgers and Y. Gu, Mobile phone-based interventions for smoking cessation. The Cochrane Library, 2012.

[54] J. Zhao, B. Freeman and M. Li, Can mobile phone apps influence people's health behavior change? An evidence review, Journal of Medical Internet Research 18(11) (2016), e287. doi:10.2196/jmir.5692. 